\title{
Estudio de patrones de metilación génica en tumores del tubo digestivo
}

\author{
Juan Carlos Roa S, Patricia García M, Angélica Melo A, \\ O scar Tapia E, Miguel Villaseca H, Juan Carlos Araya 0 , \\ Pablo G uzmán G.
}

\section{Gene methylation patterns in digestive tumors}

Background: The loss of tumor suppresor gene function damages the defensive mechanisms that protect the indemnity of genetic material. Promoter gene methylation is one of the inactivation mechanisms of suppressor genes. Aim: To study the methylation pattern of a group of genes in biopsy samples of gastrointestinal tumors. Material and methods: Forty eight gastric, 25 gallbladder, 24 colon and 6 pancreas cancer biopsy samples were randomly selected. The methylation pattern of CDH1, FHIT, CDKN2A, APC and MLH1 genes, was studied using a specific polymerase chain reaction test for methylation. Demographic, morphological and follow up variables of patients bearing the tumors were also analyzed. Results: The general methylation frequency of CDH1, FHIT, CDKN2A, APC and MLH1 genes was 64.1, 56, 39.8, 18.1 and 34\% respectively. In gastric cancer samples there was a correlation between APC gene methylation and well differentiated tumors; between CDH1 methylation and Lauren diffuse type and the presence of three or more metastasic lymph nodes; between FHIT, CDKN2A and CDH1 gene methylation and male gender. In less differentiated gallbladder tumors, the frequency of CDH1 methylation was higher. There was a tendency towards a lower survival in colon and gastric cancer when MLH1 ( $p=0.07)$ y CDKN2A ( $p=$ 0.06) were methylated, respectively. Conclusions: An abnormal methylation pattern was associated with morphological features in gastric and gallbladder cancer and with a tendency towards a lower survival in colon and gastric cancer (Rev Méd Chile 2008; 136: 451-8).

(Key words: Digestive system neoplasms; Genes, supressor; Methylation)

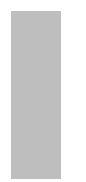

Recibido el 7 de mayo, 2007. Aceptado el 30 de octubre, 2007.

Financiado en parte por proyecto de la Dirección de Investigación de la Universidad de La Frontera (DIUFRO) 120537.

Departamento de Anatomía Patología, Laboratorio de Patología Molecular, Facultad de Medicina, Universidad de La Frontera. Temuco, Chile.

$\mathrm{L}$ os tumores del tubo digestivo son particularmente frecuentes a nivel mundial. Chile posee una de las tasas más altas de cáncer gástrico en el

Correspondencia a: Dr. Juan Carlos Roa. Departamento de A. Patológica. Laboratorio de Patología Molecular, Facultad de Medicina. Universidad de La Frontera. Manuel Montt \# 112. Código Postal 478-1176. Temuco, Chile. E mail: Jcroa@ufro.cl mundo y la más alta de cáncer de vesícula biliar ${ }^{1}$, especialmente en mujeres, donde se constituye en la primera causa de muerte por cáncer en mujeres mayores de 40 años $^{1}$. La incidencia de cáncer de colon ha presentado un continuo aumento en las últimas décadas y todo hace suponer que con el envejecimiento de nuestra población y el cambio de nuestros hábitos alimenticios, seguirá aumentado en el futuro. Otros tumores digestivos como 
cáncer de páncreas, vías biliares, esófago e hígado son menos frecuentes ${ }^{2}$.

La metilación del ADN es una modificación epigenética caracterizada por la incorporación de grupos metilos en la citosina dentro de los islotes de dinucleótidos CpG. La regulación epigenética de la función génica juega un rol relevante en el desarrollo y la metilación es el mecanismo que asegura la expresión específica de tipos celulares de un limitado grupo o espectro de genes. Diferentes enfermedades, entre ellas el cáncer, presenta patrones alterados de metilación que cambian el balance perfecto epigenético que existe en las células normales ${ }^{3}$. En las células tumorales, el patrón aberrante de metilación está caracterizado por una hipometilación global del ADN genómico y por hipermetilación de las áreas promotoras. Tales procesos conducen a inestabilidad genómica y a silenciamiento génico respectivamente $^{4-6}$. La represión transcripcional asociada a metilación aberrante de los islotes $\mathrm{CpG}$ en áreas promotoras es la consecuencia de intensos cambios de la estructura de la cromatina, producida por la interacción de metilcitosinas con diferente complejos proteicos que reclutan enzimas que modifican histonas, como las acetilasas y metilasas de histonas ${ }^{7}$.

Genes involucrados en diferentes vías metabólicas pueden sufrir metilación aberrante, incluyendo los genes reparadores de ADN, reguladores del ciclo celular, genes asociados con apoptosis, invasión celular y detoxificación celular, entre otros. Los análisis de la metilación del ADN han permitido asociar fenotipos metiladores con grupos específicos de tumores ${ }^{8}$. Estos avances han sido posibles debido a la aparición de nuevas metodologías en el campo de la investigación ${ }^{9-11}$.

El estudio de metilación génica de las áreas promotoras tiene al menos 4 potenciales aplicaciones en clínica ${ }^{12}$ : a) Identificación de células tumorales en muestras biológicas. Como los cambios en la metilación frecuentemente preceden a la aparición de tumores avanzados, su estudio puede ser usado para el diagnóstico precoz, detectando células tumorales desde muestras biológicas ${ }^{13-15}$, b) Marcador pronóstico mediante metilación de genes individuales o perfiles de metilación para grupos de genes específicos ${ }^{16-19}$, c) Marcadores de respuesta a quimioterapia u hormonoterapia ${ }^{19-23}$, d) Reactivación de genes inactivados por metilación mediante el uso de drogas demetiladoras ${ }^{24}$. La posibilidad de revertir la metilación del ADN y reactivar aquellos genes afectados es una atractiva opción para su uso como un nuevo blanco terapéutico en el tratamiento del cáncer o de lesiones preneoplásicas ${ }^{12}$. Es por esto que el estudio de la metilación génica en los tumores de nuestra población nos permitirá, probablemente, identificar blancos génicos con diferentes utilidades en el análisis tumoral.

Para nuestro estudio, se seleccionó genes supresores de tumores de diferentes vías metabólicas relacionados con el ciclo celular FHIT (Fragile Histidine Triad), CDKN2A (p16) y APC (Adenomatous Poliposis Coli), el gen MLH1 (Human Mut Homologue 1) probablemente el más importante de los genes reparadores de ADN genómico, relacionado con inestabilidad microsatelital y el gen CDH1 (Caderina E), que codifica una proteína de transmembrana que participa en el complejo de moléculas de adhesión involucrada en la metástasis e invasión tumora ${ }^{19,10,25}$.

Objetivo. Analizar el patrón de metilación de importantes genes involucrados en la carcinogénesis en pacientes con carcinomas del tubo digestivo, relacionando tales alteraciones con sus alteraciones morfológicas y el comportamiento biológico de la neoplasia.

\section{MATERIAL Y MÉTODO}

Casos. Se seleccionaron 103 casos de pacientes intervenidos quirúrgicamente por tumores digestivos: 48 de estómago (CG), 25 de vesícula biliar (CVB), 24 de colon (CC) y 6 de páncreas (CP) desde el banco de tumores del Departamento de Anatomía Patológica de la Universidad de La Frontera, todos con consentimiento informado aprobado por el Comité de Ética del Servicio de Salud Araucanía Sur. Todas las muestras fueron mantenidas en gel criopreservante (Jung, Alemania) a $-70^{\circ} \mathrm{C}$. Para la obtención del material, se seleccionó un área tumoral mediante corte de congelación y se fragmentó en cámara fría. En todos los pacientes se obtuvo seguimiento completo y las características clínicas de la serie se obtuvieron desde las fichas clínicas. Se consideró a un paciente como mapuche cuando sus 2 
apellidos correspondieron a esta etnia. Para la clasificación morfológica y etapificación de las neoplasias se utilizaron los criterios de la OMS y $\mathrm{TNM}^{26}$.

Extracción del ADN. Se realizó según protocolo del kit de aislamiento de ADN genómico Puregene (GENTRA, USA). Brevemente, los fragmentos de tejido se incubaron a $65^{\circ} \mathrm{C}$ en una solución de lisis celular y proteinasa $\mathrm{K}(1 \mathrm{mg} / \mathrm{ml})$ hasta observar lisis tisular total. Las proteínas fueron eliminadas por precipitación en acetato de amonio $7,5 \mathrm{M} \mathrm{pH}$ 7,5. El ADN fue precipitado con isopropanol, lavado con etanol 70\%, resuspendido en solución de hidratación (buffer TE pH 8,0) y almacenado a $-20^{\circ} \mathrm{C}$.

Prueba de metilación específica mediante PCR (MSP). Modificación con Bisulfito: El principio de la modificación de ADN con la técnica de bisulfito de sodio se basa en su capacidad de convertir a todos los residuos de citosina no metiladas en uracilos mediante deaminación, la citosina metilada es resistente a la reacción y permanece como citosina $^{27}$. Los iniciadores de PCR utilizados aprovechan estas diferencias para discriminar entre aquellas secuencias metiladas y no metiladas. Se usó el protocolo previamente descrito ${ }^{27}$. Brevemente, $2 \mu \mathrm{g}$ de ADN fueron denaturalizados incubando a $75^{\circ} \mathrm{C}$ por $15 \mathrm{~min}$ en un volumen de $22 \mu \mathrm{l}$ con $\mathrm{NaOH}$ (concentración final 0,27 N). Se agregó hidroquinona y bisulfito de sodio pH 5,0 preparados en fresco, a concentraciones finales de $50 \mu \mathrm{M}$ y $4,2 \mathrm{M}$, respectivamente, seguido de una incubación a $50^{\circ} \mathrm{C}$ durante $16 \mathrm{~h}$. El ADN modificado fue purificado y concentrado usando tubos Centricon YM 30 (Millipore, Bedford, MA). La desulfonación se llevó a cabo agregando $\mathrm{NaOH}$ a una concentración final de $0,3 \mathrm{~N}$ e incubando a $37^{\circ} \mathrm{C}$ durante $15 \mathrm{~min}$. Después de neutralizar la solución con acetato de amonio, se precipitó durante toda la noche a $-20^{\circ} \mathrm{C}$ con 3 volúmenes de etanol en presencia de glicógeno. Finalmente, se resuspendió en $80 \mathrm{ml}$ de agua desionizada. Amplificación del ADN: Las secuencias de partidores y condiciones de PCR han sido previamente descri$\operatorname{tas}^{28}$. Las reacciones fueron realizadas con aproximadamente 100 ng de ADN genómico modificado, 0,2 $\mu \mathrm{M}$ de cada iniciador, $200 \mu \mathrm{M}$ de cada uno de los desoxinucleótidos trifosfatos, 1,5
$\mathrm{mM}$ de $\mathrm{MgCl}$ y $0,75 \mathrm{U}$ de Taq Polimerasa (Promega) en un volumen final de $25 \mu$ l. Se usó como control negativo $100 \mathrm{ng}$ de ADN genómico sin modificar. Como control positivo de metilación se utilizó ADN genómico comercial (Promega) metilado con SssI (Biolab) y como control negativo ADN genómico (Promega) no metilado modificado. La integridad del ADN fue confirmada mediante la amplificación de secuencias no metiladas posterior a la modificación con bisulfito. Con el objetivo de verificar la eficiencia de la técnica, se secuenció $10 \%$ de las muestras que resultaron metiladas para verificar la técnica utilizada usando el sistema de secuenciación Macrogen. Las secuencias fueron analizadas y comparadas con Lasergene ${ }^{\circledR}$ v7.2 software. Los productos de PCR fueron visualizados mediante electroforesis en geles de poliacrilamida al 12\% en buffer TBE y de agarosa al $2 \%$ en buffer TAE, teñidos con bromuro de etidio (Figura 1).

Se utilizó el índice de metilación como un indicador de la proporción de regiones promotoras metiladas respecto de la totalidad de los genes estudiados y se calculó dividiendo el número de genes metilados por el número de genes analizados.

Para el análisis estadístico se consideró significación estadística con $\mathrm{p}<0,05$ y se utilizaron las pruebas de Kaplan Meier para el análisis de sobrevida Chi cuadrado y prueba de Fisher para el análisis de variables discontinuas.

\section{Resultados}

Características clínicas. Las características generales del grupo estudiado para cada tipo de tumor estudiado se observan en la Tabla 1. Este fue mayoritariamente no mapuche $(85,5 \%)$ con excepción de los carcinomas de vesícula biliar que mostraron una distribución similar al porcentaje de población mapuche de la Región de la Araucanía (aproximadamente 33\% según Censo 2002). La serie total presentó una edad promedio de 62 años. La sobrevida en promedio fue de 31 meses con rango de 1 a 108 meses. Las sobrevidas promedio de los tumores de colon y estómago alcanzaron los 39-44 meses, a diferencia de los tumores de vesícula biliar y páncreas que no superaron los 9 meses. La mayor parte de los tumores (76\%) se encontraban en etapas avanza- 


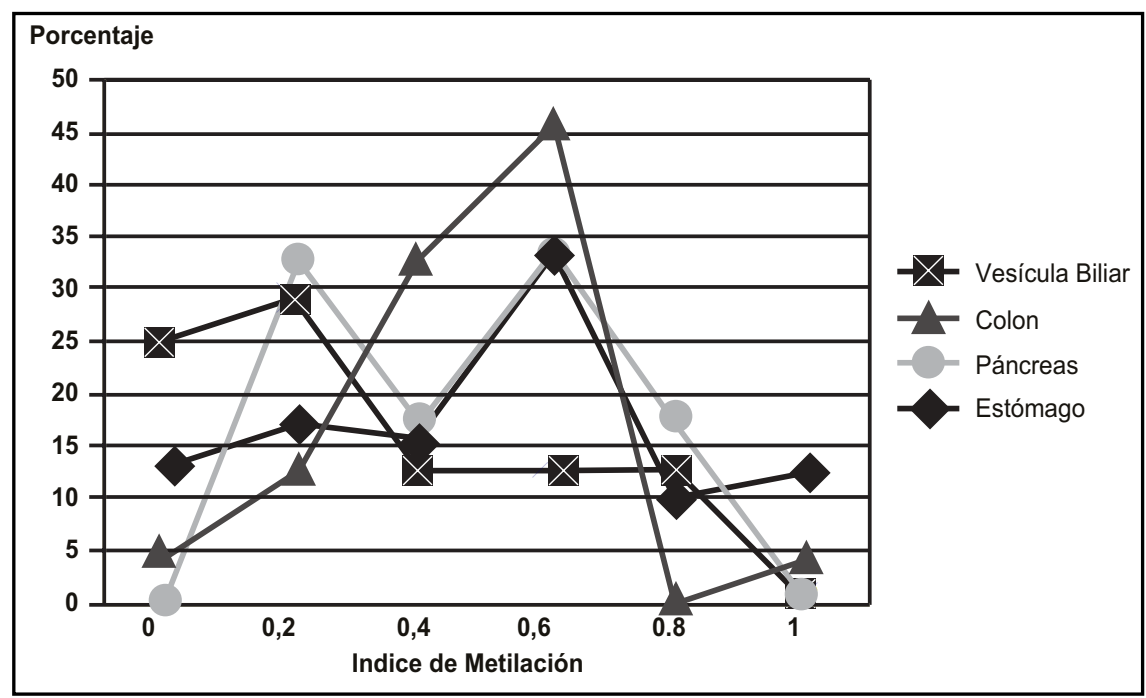

Figura 1. Índice de metilación por localización tumoral.

Tabla 1. C aracterísticas clínicas del grupo a estudiar

\begin{tabular}{|llllll|}
\hline & $\begin{array}{l}\text { Estómago } \\
(\mathrm{n}=48)\end{array}$ & $\begin{array}{l}\text { Vesícula biliar } \\
(\mathrm{n}=25)\end{array}$ & $\begin{array}{l}\text { Colon } \\
(\mathrm{n}=24)\end{array}$ & $\begin{array}{l}\text { Páncreas } \\
(\mathrm{n}=6)\end{array}$ & $\begin{array}{l}\text { Promedio } \\
(\mathrm{n}=103)\end{array}$ \\
\hline $\begin{array}{l}\text { Edad (años) } \\
\text { Sobrevida (meses) } \\
\text { Rango }\end{array}$ & 62,9 & 60,2 & 64,5 & 58,1 & 62 \\
Mapuche & 39,6 & 9 & 44,1 & 8,7 & 31 \\
$<50$ años & $4-108$ & $0-66$ & $0-124$ & $0-21$ & $1-124$ \\
TNM & $8(17 \%)$ & $8(32 \%)$ & $2(8 \%)$ & $1(17 \%)$ & $14,50 \%$ \\
I & & & $1(42 \%)$ & $2(33 \%)$ & $18,40 \%$ \\
II & $7(15 \%)$ & 0 & & & \\
III & $17(35 \%)$ & 0 & 0 & 0 & $7(7 \%)$ \\
IV & $23(48 \%)$ & $20(80 \%)$ & $15(63 \%)$ & $3(50 \%)$ & $61(60 \%)$ \\
\end{tabular}

das (etapas III y IV), especialmente los tumores de vesícula biliar y páncreas. La totalidad de los casos correspondió a adenocarcinomas.

Estado de metilación génica. Las frecuencias de metilación de la región promotora de los genes estudiados en los 103 casos de tumores del tubo digestivo analizados (Tabla 2), fluctuaron entre 18,1\% para MLH1 y 64,1\% para CDH1. Sin embargo, se observaron marcadas diferencias en el estatus de metilación de los diferentes genes en localizaciones específicas (Tabla 2). En general, las frecuencias de metilación de los órganos específicos fueron similares a los promedios encontrados para ese órgano en particular. Dentro de las diferencias más destacables para los diferentes tumores fue la alta metilación del gen de la E-caderina. El gen MLH1 presentó baja frecuencia de metilación (0-12\%) en la mayoría de los tumores, con excepción del cáncer gástrico (31\%). El cáncer de vesícula biliar fue el que presentó menor frecuencia de metilación, en la mayoría de los genes estudiados. 
Tabla 2. Frecuencia de metilación génica en los tumores estudiados

\begin{tabular}{|rlccccc|}
\hline $\mathrm{n}$ & Órgano & CDKN2A (\%) & MLH1 (\%) & FHIT (\%) & APC (\%) & CDH1 (\%) \\
\hline 48 & Estómago & 43 & 31 & 62 & 46 & 80 \\
25 & Vesícula Biliar & 20 & 4 & 32 & 32 & 60 \\
24 & Colon & 58 & 12 & 71 & 13 & 91 \\
6 & Páncreas & 33 & 0 & 83 & 33 & 83 \\
103 & Tumores digestivos & 39,8 & 18,1 & 56 & 34 & 77,8 \\
\hline
\end{tabular}

Tabla 3. Parámetros anátomo-clínicos y metilación génica específica ( $x 2$ y prueba de Kaplan M eier)

\begin{tabular}{|c|c|c|c|c|c|c|c|c|c|c|c|}
\hline \multirow{2}{*}{$\begin{array}{l}\text { Cáncer } \\
\text { gástrico }\end{array}$} & \multicolumn{2}{|c|}{ Sexo } & \multicolumn{2}{|c|}{ Metástasis ganglionar } & \multicolumn{3}{|c|}{ Grado diferenciación } & \multicolumn{2}{|c|}{ Sobrevida } & \multicolumn{2}{|c|}{ Mapuche } \\
\hline & Hombre & Mujer & Sí & No & Bien & Moderado & Poco & Muertos & Vivos & Sí & No \\
\hline FHIT & $11 / 13$ & $16 / 35^{*}$ & - & - & - & - & - & - & - & - & - \\
\hline CDKN2A & $9 / 13$ & $11 / 35^{*}$ & - & - & - & - & - & $11 / 20$ & $9 / 26^{\#}$ & - & - \\
\hline $\mathrm{CDH} 1$ & $12 / 13$ & $24 / 35^{*}$ & $7 / 11$ & $29 / 37^{*}$ & $2 / 3$ & $9 / 16$ & $25 / 29^{*}$ & - & - & - & - \\
\hline Cáncer VB & & & & & & & & & & & \\
\hline CDH1 & - & - & - & - & $0 / 1$ & $6 / 14$ & $7 / 9^{*}$ & & & - & - \\
\hline $\begin{array}{l}\text { Cáncer } \\
\text { de colon }\end{array}$ & & & & & & & & & & & \\
\hline MLH1 & - & - & - & - & - & - & - & $2 / 3$ & $1 / 21^{\$}$ & - & - \\
\hline CDH1 & - & - & - & - & - & - & - & - & - & $1 / 2$ & $21 / 22$ \\
\hline
\end{tabular}

*p $<0,05 ;$; $\mathrm{p}=0,06 ;{ }^{\$} \mathrm{p}=0,07$.

Encontramos en los casos de cáncer gástrico, que la metilación de la región promotora de los genes CDKN2A, FHIT y CDH1 fue más frecuente en hombres $(\mathrm{p}<0,05)$ (Tabla 3). En relación al grado de diferenciación del tumor, se observó que el gen CDH1 presentó una alta frecuencia de metilación en tumores poco diferenciados $(\mathrm{p}<0,05)$. En el caso del gen CDH1, se observó también que este gen estuvo frecuentemente metilado en aquellos casos que presentaron 3 o más ganglios positivos $(\mathrm{p}=0,04)$. En el cáncer de colon se evidenció una asociación de metilación mas frecuente en $\mathrm{CDH} 1$ en pacientes no mapuches $(\mathrm{p}=0,03)$. Para el cáncer de vesícula biliar se observó una tendencia no significativa $(p=0,06)$ de mayor metilación de CDH1 con menor diferenciación histológica.

El análisis del estatus de metilación entre los diferentes genes indicó que en casos de cáncer gástrico existe una correlación significativa entre gen CDKN2A metilado con respecto al estado metilado de los genes FHIT, APC y CDH1 ( $p<0,05)$. Igualmente, se observó una correlación significativa similar entre los genes CDH1 y FHIT ( $p=0,01)$.

Sobrevida y metilación. Se encontró tendencias no significativas entre la sobrevida de los pacientes con cáncer gástrico y el patrón de metilación del gen CDKN2A, y entre sobrevida con cáncer de colon y metilación del gen MLH1. El 65\% de los casos presentaron más de 2 genes metilados, es decir, un índice de metilación de 0,4 o superior (Figura 2).

\section{DisCUSIÓN}

En nuestro estudio analizamos el perfil de metilación de 5 conocidos genes, todos ellos relacionados con distintas vías carcinogenéticas alteradas en tumores 


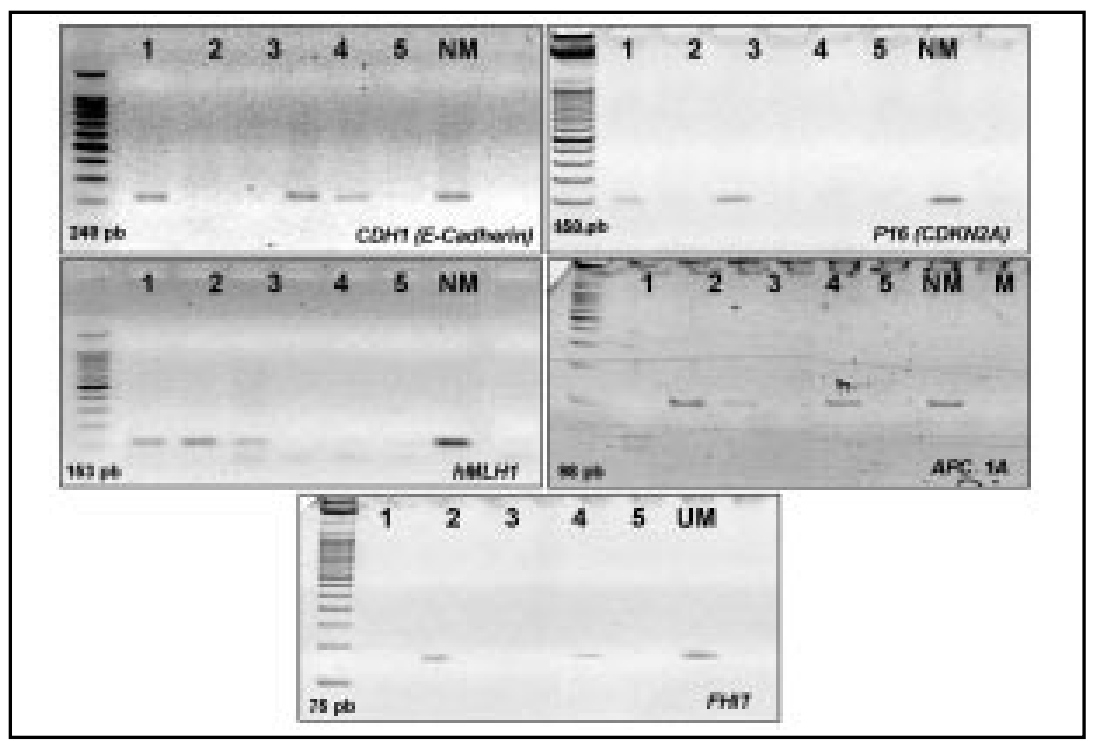

Figura 2. Fotografía compuesta de geles de agarosa que muestra el resultado del análisis de metilación en los 5 genes estudiados donde se puede observar el tamaño variable de las bandas amplificadas. $\mathrm{M}=$ control positivo ADN genómico metilado y modificado, $\mathrm{NM}=$ control negativo $\mathrm{ADN}$ genómico no metilado y modificado y $\mathrm{B}=\mathrm{Blanco}$.

humanos. La prueba aplicada en este estudio, metilación específica mediante PCR (MSP), detecta hasta $0,1 \%$ de ADN metilado, constituyéndose en una técnica altamente sensible que permite la pesquisa de este fenómeno, incluso en las primeras etapas del desarrollo carcinogenético, donde la cantidad de alelos metilados puede ser muy escasa ${ }^{27}$.

Los tumores de la vesícula biliar presentaron una frecuencia de genes moderadamente metilados, sin embargo, notablemente más bajos que en los otros tumores estudiados. En este mismo sentido, el índice de metilación, relación que representa la cantidad de genes metilados sobre el número de genes estudiados, mostró que sólo un tercio de las muestras de CVB mostraron $40 \%$ o más de los genes metilados (IM $\geq 0,4)$ y en el resto de los tumores, éste alcanzaba a más de los $2 / 3$. La causa de esta diferencia no es clara, no obstante la influencia de la inflamación crónica o contacto con diferentes carcinógenos debiese ser investigado.

Aun cuando la metilación del gen CDH1 es muy frecuente en todos los tumores estudiados, cuando se excluye a los CVB, la frecuencia de metilación se eleva hasta $85 \%$ en el resto de los tumores digestivos. El gen CDH1 (Caderina E) pertenece a una familia de genes que se encuentran directamente relacionados con procesos de invasión tumoral y desestabilización del citoesqueleto y se han asociado a tumores menos diferenciados y que presentan clara relación con pronóstico ${ }^{29-33}$. Eso explica y valida nuestros resultados en cáncer gástrico y CVB donde se puede evidenciar una directa asociación entre el grado de diferenciación tumoral (CVB y CG), metástasis ganglionares linfáticas (CG) y metilación génica. Es destacable la asociación de etnia mapuche y metilación de $\mathrm{CDH} 1$, lo cual no tiene una explicación consistente en este momento.

El gen CDKN2A es un gen supresor de tumores que codifica una proteína conocida como p16, que es un inhibidor de una ciclina dependiente de quina$\mathrm{sa}^{34,35}$. Una menor expresión de p16 resulta en un aumento de la actividad de la ciclina $\mathrm{D}$ dependiente de quinasa, lo cual se traduce en una fosforilación aberrante del gen retinoblastoma (RB), con un subsecuentemente aceleramiento del crecimiento celular ${ }^{36,37}$, favoreciendo la aparición de una neoplasia. En nuestro estudio, el gen CDKN2A mostró una particular predisposición a estar metilado en pacientes con cáncer gástrico de sexo masculino, y en pacientes con peor sobrevida. Sorprendentemente, la metilación de este gen se asoció significativamente con la presencia de metilación en FHIT, APC y CDH1. Estas vías carcinogénicas se encuentran separadas de la vía 
del CDKN2A, por lo que subsecuentes estudios mecanísticos deberían dilucidar la posible relación que pudiese existir entre ellos.

El gen MLH1 clásicamente asociado a inestabilidad microsatelital, se ha correlacionado con el pronóstico en tumores gástricos, de colon y recto y algunos tumores endocrinos pancreáticos ${ }^{38,39}$.

El gen FHIT, inicialmente de función desconocida, se reconoce con certeza su función supresora de tumores y ha mostrado una clara tendencia a estar metilado en subgrupos de pacientes con peor pronóstico ${ }^{40}$.

La inactivación del gen APC se ha correlacionado con peor pronóstico ${ }^{41}$. En nuestra serie no observamos relación del estatus de metilación de estos genes con características clínico-patológicas.

La alta frecuencia de metilación anormal en pacientes con tumores digestivos confirma la importancia de este tipo de alteración epigenética en la carcinogénesis de estas neoplasias. Confirmamos la

\section{REFERENCIAS}

1. Medina E, Kaempffer AM. [Cancer mortality in Chile: epidemiological considerations]. Rev Méd Chile 2001; 129: 1195-202.

2. Medina E, Kaempffer AM. [Adult mortality in Chile]. Rev Méd Chile 2000; 128: 1144-9.

3. BIRD AP. CPG-rich islands and the function of DNA methylation. Nature 1986; 321: 209-13.

4. Jones PA, LaIRD PW. Cancer epigenetics comes of age. Nat Genet 1999; 21: 163-7.

5. Plass C. Cancer epigenomics. Hum Mol Genet 2002; 11: 2479-88.

6. Esteller M, Herman JG. Cancer as an epigenetic disease: DNA methylation and chromatin in human tumours. J Pathol 2002; 196: 1-7.

7. Esteller M. CpG island methylation and histone modifications: biology and significance. Ernst Schering Res Found Workshop 2006; 115-26.

8. EsTeLLeR M. Epigenetics provides a new generation of oncogenes and tumour- genes. Br J Cancer 2006; 94: $179-83$.

9. Issa JP. CpG island methylator phenotype in cancer. Nat Rev Cancer 2004; 4: 988-93.

10. Paz MF, Fraga MF, Avila S, Guo M, Pollan M, Herman JG, ET AL. A systematic profile of DNA methylation in human cancer cell. Cancer Res 2003; 63: 1114-21.

11. Esteller M, Sparks A, Toyota M, Sanchez-Cespedes M, aparición de diferentes perfiles de metilación específicos de las áreas promotoras génicas, con una clara disminución de los niveles de metilación en los tumores vesiculares en relación con los otros tumores gastrointestinales. De nuestro análisis, podemos comprobar que la metilación de estos genes se relacionó en forma estadísticamente significativa con importantes parámetros clínicos y morfológicos asociados con superviviencia para tumores específicos como fueron la presencia de metástasis ganglionar linfática en cáncer gástrico (CDH1) y el grado de diferenciación histológica en cáncer gástrico y de vesícula biliar (CDH1). Mostrando, además, tendencias no significativas entre cáncer gástrico, de colon y el estatus de metilación de CDKN2A y MLH1 respectivamente. Así, el hallazgo de alteraciones en el patrón de metilación de genes supresores de tumores en tumores digestivos es una herramienta más en la búsqueda de nuevos criterios de decisión terapéutica e indicadores pronósticos.

Capella G, Peinado MA, et al. Analysis of adenomatous polyposis coli promoter hypermethylation in human cancer. Cancer Res 2000; 60: 4366-71.

12. ROA JC. [Genetic methylation in carcinogenesis and its application in oncology]. Med Clin (Barc) 2006; 126: 455-6.

13. Lee WH, Morton Ra, Epstein JI, Brooks JD, Campbell PA, Bova GS ET AL. Cytidine methylation of regulatory sequences near the pi-class glutathione Stransferase gene accompanies human prostatic carcinogenesis. Proc Natl Acad Sci USA 1994; 91: 11733-7.

14. Jerónimo C, Usadel H, Henrique R, Oliveira J, Lopes C, Nelson WG et al. Quantitation of GSTP1 methylation in non-neoplastic prostatic tissue and organ-confined prostate adenocarcinoma. J Natl Cancer Inst 2001; 93: 1747-52.

15. Esteller M, Corn PG, Baylin SB, Herman JG. A gene hypermethylation profile of human cancer. Cancer Res 2001; 61: 3225-9.

16. Alaminos M, Davalos V, Ropero S, Setien F, Paz MF, HeRrAnz M ET al. EMP3, a myelin-related gene located in the critical 19q13.3 region, is epigenetically silenced and exhibits features of a candidate tumor suppressor in glioma and neuroblastoma. Cancer Res 2005; 65: 2565-71.

17. Alaminos M, Davalos V, Cheung NK, Gerald WL, ESTELLeR M. Clustering of gene hypermethylation 
associated with clinical risk groups in neuroblastoma. J Natl Cancer Inst 2004; 96: 1208-19.

18. Esteller M, Gaidano G, Goodman SN, Zagonel V, Capello D, Botto B ET al. Hypermethylation of the DNA repair gene O(6)-methylguanine DNA methyltransferase and survival of patients with diffuse large B-cell lymphoma. J Natl Cancer Inst 2002; 94: 26-32.

19. Esteller M. CpG island hypermethylation and tumor suppressor genes: a booming present, a brighter future. Oncogene 2002; 21: 5427-40.

20. Esteller M, Herman JG. Generating mutations but providing chemosensitivity: the role of O6-methylguanine DNA methyltransferase in human cancer. Oncogene 2004; 23: 1-8.

21. Plumb Ja, Strathdee G, Sludden J, Kaye SB, Brown $R$. Reversal of drug resistance in human tumor xenografts by 2'-deoxy-5-azacytidine-induced demethylation of the hMLH1 gene promoter. Cancer Res 2000; 60: 6039-44.

22. Tew KD, Ronai Z. GST function in drug and stress response. Drug Resist Updat 1999; 2: 143-7.

23. Ottaviano YL, Issa JP, Parl FF, Smith HS, Baylin SB, Davidson NE. Methylation of the estrogen receptor gene CpG island marks loss of estrogen receptor expression in human breast cancer cells. Cancer Res 1994; 54: 2552-5.

24. Wijermans P, Lubbert M, Verhoef G, Bosly A, Ravoet C, Andre M et al. Low-dose 5-aza-2'-deoxycytidine, a DNA hypomethylating agent, for the treatment of high-risk myelodysplastic syndrome: a multicenter phase II study in elderly patients. J Clin Oncol 2000; 18: 956-62.

25. Wajed SA, Laird PW, DeMeester TR. DNA methylation: an alternative pathway to cancer. Ann Surg 2001; 234: 10-20.

26. The new TNM classification in gastroenterology (1997). Endoscopy 1998; 30: 643-9.

27. Herman JG, Graff JR, Myohanen S, Nelkin BD, BaYLIN SB. Methylation-specific PCR: a novel PCR assay for methylation status of CpG islands. Proc Natl Acad Sci USA 1996; 93: 9821-6.

28. Roa JCA, L Tapia, O Martínez, J Araya, JC Villaseca, M RoA, I. Patrón de metilación génico en cáncer de mama. Rev Méd Chile 2004; 132: 1069-77.

29. Widschwendter A, Ivarsson L, Blassnig A, Muller HM, Fiegl H, Wiedemair A et Al. CDH1 and CDH13 methylation in serum is an independent prognostic marker in cervical cancer patients. IntJ Cancer 2004; 109: 163-6
30. Graziano F, Arduini F, Ruzzo A, Bearzi I, Humar B, More H, ET AL. Prognostic analysis of E-cadherin gene promoter hypermethylation in patients with surgically resected, node-positive, diffuse gastric cancer. Clin Cancer Res 2004; 10: 2784-9.

31. Maruyama R, Toyooka S, Toyooka KO, Virmani AK, Zochbauer-Muller S, Farinas AJ et al. Aberrant promoter methylation profile of prostate cancers and its relationship to clinicopathological features. Clin Cancer Res 2002; 8: 514-9.

32. Maruyama R, Toyooka S, Toyooka KO, Harada K, Virmani AK, Zochbauer-Muller S, et al. Aberrant promoter methylation profile of bladder cancer and its relationship to clinicopathological features. Cancer Res 2001; 61: 8659-63.

33. Dulaimi e, Uzzo RG, Greenberg RE, Al-Saleem T, CAIRns P. Detection of bladder cancer in urine by a tumor suppressor gene hypermethylation panel. Clin Cancer Res 2004; 10: 1887-93.

34. SHerr CJ. The Pezcoller lecture: cancer cell cycles revisited. Cancer Res 2000; 60: 3689-95.

35. Palmero I, Peters G. Perturbation of cell cycle regulators in human cancer. Cancer Surv 1996; 27 : 351-67.

36. Schafer KA. The cell cycle: a review. Vet Pathol 1998; 35: 461-78.

37. Hruban RH, Iacobuzio-Donahue C, Wilentz RE, GogGins M, KeRn SE. Molecular pathology of pancreatic cancer. Cancer J 2001; 7: 251-8.

38. House MG, Herman JG, Guo MZ, Hooker CM, Schulick RD, Cameron JL et al. Prognostic value of hMLH1 methylation and microsatellite instability in pancreatic endocrine neoplasms. Surgery 2003; 134: 902-8; discussion 909.

39. Goel A, Arnold CN, Niedzwiecki D, Carethers JM, Dowell JM, Wasserman L et al. Frequent inactivation of PTEN by promoter hypermethylation in microsatellite instability-high sporadic colorectal cancers. Cancer Res 2004; 64: 3014-21.

40. Maruyama R, Sugio K, Yoshino I, Maehara Y, Gazdar AF. Hypermethylation of FHIT as a prognostic marker in nonsmall cell lung carcinoma. Cancer 2004; 100: 1472-7.

41. Kawakami K, Brabender J, Lord RV, Groshen S, Greenwald BD, Krasna MJ et al. Hypermethylated APC DNA in plasma and prognosis of patients with esophageal adenocarcinoma. J Natl Cancer Inst 2000; 92: 1805-11. 